\section{A report of the 2009-2011 paternity and rela- tionship testing workshops of the English Speaking Working Group of the International Society For Forensic Genetics}

Participation in proficiency testing has gained more interest as more laboratories have become accredited. Since 1991, the English Speaking Working Group (ESWG) of the International Society for Forensic Genetics (ISFG) has offered an annual exercise/workshop [1-5]. In 2010, the workshop changed name from Paternity Testing Workshop to Relationship Testing Workshop. The number of participating laboratories in the Paternity Testing Workshop has increased from 9 in 1991 to 69 in 2007 [1,5]. In the period from 2009 to 2011 the Paternity or Relationship Testing Workshop has reached a plateau with 62 participants each year (Supplementary Appendix A). The results of the workshops were analysed and presented at the annual ESWG meetings (Supplementary Appendix B). The workshop is divided into three parts. The first part is the paternity or relationship testing exercise. Here, the participating laboratories are to perform genetic testing according to usual protocols on blood samples from a fictive relationship case. The second part of the workshop is a questionnaire, which enables comparison of laboratory strategies and methods. The third part is a paper challenge, where laboratories are invited to perform biostatistical calculations. All protocols were approved by the Danish ethical committee (KF-01037/93 and H-1-2011-598 081).

Supplementary material related to this article can be found, in the online version, at doi:10.1016/j.fsigen.2013.06.004.

The Paternity Testing Commission (PTC) of the ISFG recommends that paternity testing is performed in accordance with the ISO 17025 standard that specifies general requirements for laboratories for competent performance of testing [6]. The fraction of accredited laboratories has increased during the last 10 years from $46 \%$ in 2002 [5] to $73 \%$ in 2011 . In $2011,87 \%$ of the accredited laboratories were accredited according to the ISO17025 standard. Autosomal STR kits were available in all participating laboratories (Supplementary Table 1). The use of new autosomal STR multiplex kit, with five additional loci $[7,8]$ included in the European Standard Set (ESS) was reported for the first time in 2011. The new STR kits were used by 55\% of the laboratories. In $2011,8 \%$ of the laboratories reported the use of the newly released Investigator DIPplex PCR amplification kit (Qiagen) for analysis of 30 biallelic autosomal insertion/deletion polymorphisms. Analysis of insertion/deletion polymorphisms offers an alternative or supplement to STR-based and SNP-based relationship testing [9-11]. X-STR analysis and mtDNA sequencing was available as an additional test in approximately one third of the laboratories, whereas SNP analysis is still not commonly used. Y-STR analysis was available in more than $80 \%$ of the laboratories. There was tendency towards the use of commercial kits for both autosomal and gonosomal STR systems.
Supplementary material related to this article can be found, in the online version, at doi:10.1016/j.fsigen.2013.06.004.

A total of $96-100 \%$ of the participating laboratories reported biostatistical evidential values either as a PI or W-value. There was a high variation in the requirement for issuing a report with sufficient positive weight for paternity. However, the majority of laboratories required a PI of more than 10,000 corresponding to a probability of more than $99.99 \%$, assuming a priori probability of paternity $=0.5$ (Supplementary Table 2). One third of the laboratories required a PI threshold below 10,000, even though kits for typing at least 15 autosomal STR systems were used. Hence, the required PI threshold cannot only be explained by the number of tested polymorphic STR markers. On the other hand, there was uniformity in the criteria for paternity exclusion with a certain number of inconsistencies as the preferred requirement, typically based on at least 15 investigated STR systems. The preference of the Paternity Testing Commission of ISFG is to use an exclusion criterion in terms of a LR threshold [12]. Such an exclusion criterion was used by $11 \%$ of the laboratories in 2011. Most laboratories used computer software for biostatistical calculations (Supplementary Table 3). The use of computer software for biostatistical calculations has remained fairly constant since 2009. The number of laboratories using the software Familias [13] has increased.

Supplementary material related to this article can be found, in the online version, at doi:10.1016/j.fsigen.2013.06.004

The submitted typing results of the paternity and relationship testing workshops 2009-2011 showed an increasing degree of concordance concerning methods and applied systems. The observed discrepancies in 2009-2011 counted for $0.04 \%-0.14 \%$ of all STR allele results (Supplementary Fig. 1). The tendency of using the same commercial kits for testing the blood samples probably accounts for some of the uniformity in the obtained typing results. The laboratories generally agreed on their conclusions of the paternity testing exercises. The only discrepancy was seen in the 2011 exercise, where the laboratories were to investigate if two siblings were half or full siblings. Only $46 \%$ of the laboratories typing autosomal STR and Y STR systems concluded that the results were in favour of half siblings. In contrast, $87 \%$ of the laboratories also typing mtDNA, X-STR systems or autosomal SNPs concluded that the results were in favour of half siblings. Hence, in this type of deficiency case, the use of additional markers, especially the X-chromosomal and mtDNA markers, was informative to disclose the genetic relationship.

Supplementary material related to this article can be found, in the online version, at doi:10.1016/j.fsigen.2013.06.004.

The paper challenges (Supplementary Appendix C) showed a considerable variation in the formulas used among the participants when rare alleles, genetic inconsistencies or possible silent alleles were present and for biostatistical calculations based on haplotypes (Supplementary Tables 4 and 5). Most laboratories did consider the possibility of mutational events. However, a large number of different formulas were used for calculating the LR 
leading to considerable variation in the total LRs. Silent alleles arise when the allele size is outside the range of measurement or, when mutations in primer-binding sites have occurred. Only few participants followed the recommendation from the Paternity Testing Commission of the ISFG to consider the possibility of silent alleles when only one allele is observed [12]. The majority of the laboratories consider silent alleles in cases of opposite homozygosity between the parent and the child. Furthermore, there was a high variability in how the participants calculated the probability of a rare allele. Finally, various approaches were utilised for calculation of X-STR systems resulting in different LR values. When omitting the more complicated genetic events and X-STR, Y-STR and mtDNA data, there was a general high uniformity in how the statistics were calculated.

Supplementary material related to this article can be found, in the online version, at doi:10.1016/j.fsigen.2013.06.004.

\section{References}

[1] D. Syndercombe Court, P. Lincoln, A review of the 1991-1994 Paternity Testing Workshops of the English Speaking Working Group, Adv. Forensic Haemogenet. 6 (1996) 683-685.

[2] A. Bjerre, D.S. Court, P. Lincoln, N. Morling, A report of the 1995 and 1996 Paternity Testing Workshops of the English Speaking Working Group of the International Society for Forensic Haemogenetics, Forensic Sci. Int. 90 (1997) 41-55.

[3] C. Hallenberg, N. Morling, A report of the 1997, 1998 and 1999 Paternity Testing Workshops of the English Speaking Working Group of the International Society for Forensic Genetics, Forensic Sci. Int. 116 (2001) 23-33.

[4] C. Hallenberg, N. Morling, A report of the 2000 and 2001 paternity testing workshops of the English speaking working group of the international society for forensic genetics, Forensic Sci. Int. 129 (2002) 43-50.

[5] A.R. Thomsen, C. Hallenberg, B.T. Simonsen, R.B. Langkjaer, N. Morling, A report of the 2002-2008 paternity testing workshops of the English speaking working group of the International Society for Forensic Genetics, Forensic Sci. Int. Genet. 3 (2009) 214-221.

[6] N. Morling, R.W. Allen, A. Carracedo, H. Geada, F. Guidet, C. Hallenberg W. Martin, W.R. Mayr, B. Olaisen, V.L. Pascali, P.M. Schneider, Paternity Testing Commission of the International Society of Forensic Genetics: recommendations on genetic investigations in paternity cases, Forensic Sci. Int. 129 (2002) 148-157.
[7] P. Gill, L. Fereday, N. Morling, P.M. Schneider, New multiplexes for Europeamendments and clarification of strategic development, Forensic Sci. Int. 163 (2006) 155-157

[8] P. Gill, L. Fereday, N. Morling, P.M. Schneider, The evolution of DNA databases recommendations for new European STR loci, Forensic Sci. Int. 156 (2006) 242 244.

[9] A.M. Neuvonen, J.U. Palo, M. Hedman, A. Sajantila, Discrimination power of Investigator DIPplex loci in Finnish and Somali populations, Forensic Sci. Int. Genet. (2011)

[10] B.L. Larue, J. Ge, J.L. King, B. Budowle, A validation study of the Qiagen Investigator DIPplex(R) kit; an INDEL-based assay for human identification, Int. J. Legal Med. (2012).

[11] S.L. Friis, C. Borsting, E. Rockenbauer, L. Poulsen, S.F. Fredslund, C. Tomas, N. Morling, Typing of 30 insertion/deletions in Danes using the first commercial indel kit - Mentype(R) DIPplex, Forensic Sci. Int. Genet. 6 (2012) e72-e74.

[12] D.W. Gjertson, C.H. Brenner, M.P. Baur, A. Carracedo, F. Guidet, J.A. Luque, R. Lessig, W.R. Mayr, V.L. Pascali, M. Prinz, P.M. Schneider, N. Morling, ISFG: recommendations on biostatistics in paternity testing, Forensic Sci. Int. Genet. 1 (2007) 223-231.

[13] T. Egeland, P.F. Mostad, B. Mevag, M. Stenersen, Beyond traditional paternity and identification cases. Selecting the most probable pedigree, Forensic Sci. Int. 110 (2000) 47-59

Lena Poulsen* Susanne Lunøe Friis Charlotte Hallenberg Bo Thisted Simonsen Niels Morling Section of Forensic Genetics, Department of Forensic Medicine, Faculty of Health and Medical Sciences, University of Copenhagen, 11 Frederik V's Vej, DK-2100 Copenhagen, Denmark

*Corresponding author. Tel.: +45 3532 6314; fax: +45 35326270 E-mail address: lena.poulsen@sund.ku.dk (L. Poulsen)

29 April 2013 